\title{
Understanding L.O.V.E. (Left Out Variables Error): A Method for Estimating the Effects of Omitted Variables
}

\author{
Robert Mauro \\ University of Oregon
}

\begin{abstract}
Whenever nonexperimental methods are used to test a hypothesis and 1 or more predictor (independent) variables that may affect the criterion (dependent) variable are omitted from the analyses, it is possible that the estimates of the effects of the predictors are biased or that the omitted variable could account entirely for the effects attributed to one or more of the predictors. In this article, a technique is developed for determining when a variable omitted from a linear model can account for the effects attributed to a predictor included in that model.
\end{abstract}

Social scientists are rarely able to obtain information on all of the factors that may affect their criterion (outcome, dependent) variables. Whenever researchers use nonexperimental methods and fail to account for all of the variables that affect a criterion, their inferences about the effects of the predictors (independent variables) on that criterion may be biased. ${ }^{1}$ Whenever a relevant variable is neither held constant nor entered into an analysis, that variable could account entirely for the effects attributed to one or more of the predictor variables included in the analysis. In this article, a technique is developed for determining when a variable omitted from a linear model can account for the effects attributed to a predictor included in that model. This technique is designed to provide a general indication of the potential effects of an omitted variable. There is no associated statistical distribution theory.

For an omitted variable to account for the effect of a specific predictor, the omitted variable must (a) have a substantial effect on the criterion variable, (b) be substantially correlated with the predictor in question, and (c) not be substantially correlated with the other predictors in the model.

For example, a clinical psychologist might theorize that a disorder is the result of a genetic aberration, whereas another researcher theorizes that this disorder is the result of sociocultural factors and a high level of life stress. The first investigator might then conduct a study in which the family histories of patients are examined and conclude that controlling for age, sex, and socioeconomic status, family history (reflecting the genetic component) of the disorder is a significant predictor of the disorder. The second researcher could criticize this research for not including measures of other potentially important variables, such as life stress, and hypothesize that had a measure of

The comments and suggestions made by Jennifer Alansky Mauro, Richard Berk, Bill Chaplin, Robyn Dawes, Phoebe Ellsworth, Lewis Goldberg, Samuel Gross, Myron Rothbart, Associate Editor Larry Hedges, and two anonymous reviewers on earlier versions of this article are greatly appreciated.

Correspondence concerning this article should be addressed to Robert Mauro, Department of Psychology, University of Oregon, Eugene, Oregon 97403-1227. life stress been included in the analysis, the effect attributed to family history would disappear.

To determine whether this is a reasonable criticism, one must ask to what extent life stress is correlated with (a) having the disorder, (b) having a family history of the disorder, and (c) age, sex, and socioeconomic status. All of these correlations are important. If life stress has no effect on having the disorder, then its omission from the analysis will not bias the estimate of the effect of family history. If life stress is related to having the disorder but is not correlated with having a family history of the disorder, its omission will again not bias the estimate of the effect of family history. ${ }^{2}$ If life stress is related to both having the disorder and having a family history of the disorder, and it is substantially correlated with age, sex, or socioeconomic status (e.g., life stress may be related to poverty), then its omission again will have little influence on the estimate of the effect of family history (because the effect of life stress on developing the disorder will be "carried" by one or more of the other predictors). Only if life stress is predictive of having the disorder, substantially correlated with having a family history of the disorder, and not substantially correlated with the other predictors will its omission seriously bias the estimate of the effect attributed to family history.

In many cases, it is impossible to determine how variance in a criterion should be divided between correlated predictors (cf. Meehl, 1971; Pedhazur, 1982). For instance, in the above example one could argue that even if life stress could account for some of the variance in the occurrence of the disorder that was attributed to genetic factors, only that portion of the variance in the disorder not attributable to genetic factors should be attributed to life stress (perhaps because the subjects had genes before they had stress). If this argument were accepted, then the omission of life stress from the analysis would not be a serious problem. However, one could make a similar argument for the

\footnotetext{
' In true experiments, the random assignment of subjects to conditions ensures that all of the variables not under experimental control are uncorrelated with those that are.

${ }^{2}$ Omitting a relevant variable will, however, increase the unexplained variance in the criterion.
} 
priority of life stress over genetic inheritance as a causal factor (perhaps based on temporal proximity of the cause to the effect).

The hypothesis that an omitted variable could account for the effect attributed to a predictor included in an analysis is predicated on the assumption that the variance in the criterion "explained" by both variables should be attributed to the omitted variable, or at least shared between them. ${ }^{3}$ The methods described in this article are useful primarily when one is willing, at least for the sake of argument, to accept this assumption and examine the hypothesis that a predictor has an effect on the criterion above that attributable to other variables-both those included in and those omitted from the analysis. In this case, the estimate of the effect of a predictor obtained from a model lacking a variable may be considered to be "biased" compared with the estimate that would be obtained from a model containing the omitted variable as well as the predictors included in the analysis.

\section{Left Out Variables Error}

The error introduced into an analysis by the omission of a relevant variable is called "omitted variables error" or "left out variables error." It is one of a class of potential sources of error that are termed "misspecification errors" (for general discussions, see Leamer, 1978; Mariano \& Ramage, 1983; and Ramsey, 1974).

A statistical model is said to be misspecified when the variables included in the model or the specified relations between the variables differ from those of the "true" model. In practice, researchers rarely know the "true" model. Hence, most regression models are misspecified to some degree, and one can only estimate the extent to which possible misspecifications may have biased the obtained parameter estimates. Procedures for testing and dealing with many types of misspecification error, including the inclusion of irrelevant variables (cf. Kennedy, 1985; Pedhazur, 1982) and the misspecification of the functional form of the model (cf. Pedhazur, 1982; Ramsey, 1969, 1974), have been developed.

The omission of relevant variables is the most difficult type of misspecification error to detect and the one with the most severe ramifications. The omission of a relevant variable will inflate the estimates of the standard errors of the included variables, causing the tests of significance for these predictors to be inaccurate. The constant term will also be biased (unless the mean of the observations on the omitted variable happened to be zero). Whenever the omitted variable is correlated with the included variables, the estimates of the regression coefficients for these variables will be biased (see below).

When a relevant variable is omitted from a model, some of the effect of that variable will be incorporated into the residual error of the model that is analyzed. If some variable or variables can be used as a proxy for the omitted variable, a test of the "net" effect of the omitted variable can be conducted by examining the relation between this variable or set of variables and the residuals from the analyzed model. Ramsey (1969; Ramsey \& Schmidt, 1976) has developed a procedure (RESET, an acronym for REgression Specification Error Test) for making this test by adding the proxy variable(s) to the variables included in the model and then testing the coefficients of the proxy set against zero using an $F$ test. Of course, if useful proxy variables had been available, they probably would have been included in the original analysis. ${ }^{4}$ Therefore, a linear combination of the predictors following some transformation (e.g., raising to a power) is frequently used to create the proxy variable(s) needed for the RESET procedure (Ramsey, 1969; Thursby \& Schmidt, 1977). The hope is that this new variable will add important information to the model that is not represented by the original predictors. However, the effect of the omitted variable frequently cannot be approximated by a linear combination of the included predictors, making this substitution inappropriate. Furthermore, the effect of the omitted variable on the residuals is frequently small when the omitted variable is highly correlated with the included predictors. Thus, the "net" effect of the omitted variable, given the other predictors, may be small when the biasing effect of the omission is great.

Instead of attempting to measure the net effect of an omitted variable, several authors have suggested measuring the sensitivity of the estimates of the effects of variables included in an analysis to the potentially biasing effects of an omitted variable. Rosenbaum and Rubin (e.g., Rosenbaum, 1984, 1986; Rosenbaum \& Rubin, 1983; Rubin, 1973) have concentrated on developing a technique for estimating the average effect of a "treatment" on a binary or continuous criterion variable when a relevant predictor has been omitted from the analysis. In essence, their technique uses estimates of the average differences between treatment groups on the omitted variable and an estimate of the coefficient for the regression of the omitted variable on the criterion variable given the included predictors to obtain adjusted estimates of the treatment effect. Because good estimates of the average differences on the omitted variable and the coefficient of the omitted variable are frequently difficult to obtain, Rosenbaum and Rubin (1983) suggested examining the effects of a range of possible values of these quantities. Unfortunately, when the omitted variable is correlated with the predictors included in the analysis, it is very difficult to estimate what the regression coefficient of the omitted variable would be. In models with correlated predictors, the coefficient for a predictor may take on nonintuitive values-sometimes opposite in sign to the simple correlation of the predictor with the criterion variable (for relevant discussions of this problem, see Cohen \& Cohen, 1983; and Pedhazur, 1982).

Instead of relying on estimates of the regression coefficient of the omitted variable, the procedures proposed below for estimating the effect of the omitted variable on the estimate of the treatment effect use estimates of the simple correlations among the omitted, criterion, and predictor variables. Reichardt and

\footnotetext{
${ }^{3}$ It is also necessary that the omitted variable be a measure of a factor not already included in the model. As the correlation between the omitted variable and a predictor approaches unity, the model will become unestimable, and it will be impossible to "separate" the effects of the two variables.

${ }^{4}$ If another variable is used as a proxy for the omitted variable, one could treat the proxy as an indicator of the omitted variable measured with error. See Klepper and Leamer (1984) for an interesting discussion of working with regression models in which the variables are measured with error.
} 
Gollob $(1986,1987)$ independently suggested an approach to estimating the bias due to an omitted variable similar to the first technique discussed below. These authors noted that when the necessary correlations can be estimated, limits can be set on the possible bias caused by an omitted variable. This approach is directly applicable to all regression models, including the analysis of covariance models discussed by Rosenbaum and Rubin (1983; Rosenbaum, 1986).

\section{Describing the Effect of Omitting a Variable}

To determine more precisely the extent to which the omission of a variable alters the effects attributed to a predictor, one must examine how the effects attributed to that predictor are altered by changes in the correlations between the omitted variable and (a) the criterion variable, (b) the predictor in question -here called the primary predictor, and (c) the other predictors included in the model-here called covariates.

The effect of an omitted variable $\left(X_{o}\right)$ on inferences about the effect of a predictor $\left(X_{p}\right)$ can be seen easily in a linear regression on two standardized variables. ${ }^{5}$ In this case, the linear model is

$$
Y=B_{p} X_{p}+B_{o} X_{o}+e,
$$

where the $B_{i}$ are standardized regression coefficients. The ordinary least squares regression coefficients are

$$
B_{p}=\frac{r_{y p}-r_{y o} r_{p o}}{1-r_{p o}^{2}} \quad B_{o}=\frac{r_{y o}-r_{y p} r_{p o}}{1-r_{p o}^{2}}
$$

where the $r_{i j}$ are the simple correlations between variable $i$ and variable $j$ and $r_{i j}{ }^{2}$ is the square of this simple correlation.

If a researcher failed to include $X_{o}$ when examining the effect of $X_{p}$ on $Y$, the coefficient associated with $X_{p}\left(B_{p}\right)$ would equal $r_{y p}$, the simple correlation of $X_{p}$ with $Y$. Thus, if $Y$ were regressed on $X_{p}$ alone, the coefficient associated with $X_{p}$ would be biased when the correlation between the omitted variable and the criterion variable $\left(r_{y_{0}}\right)$ and the correlation between the omitted variable and the primary predictor $\left(r_{o p}\right)$ are nonzero. If $X_{p}$ is uncorrelated with $X_{0}$ (i.e., $r_{p o}=0$ ), omitting $X_{0}$ will not bias the estimate of the effect of $X_{p}$ on $Y$. Only when the omitted variable is correlated with the primary predictor will its omission bias the estimates obtained. The larger the correlation of the omitted variable with the criterion, the larger this bias will be.

When more than two predictors are included in a model, the correlations between the omitted variable and the covariates must also be considered. The three-predictor case can be used to illustrate this effect. The linear model for this regression on standardized variables is

$$
Y=B_{p} X_{p}+B_{i} X_{i}+B_{o} X_{o}+e .
$$

The regression coefficient associated with the primary predictor $\left(X_{p}\right)$ in this model is

$$
B_{p}=\frac{r_{y p}\left(1-r_{i o}{ }^{2}\right)+r_{y i}\left(r_{p o} r_{i o}-r_{p i}\right)+r_{y o}\left(r_{p i} r_{i o}-r_{p o}\right)}{1+2 r_{p i} r_{i o} r_{p o}-r_{p o}{ }^{2}-r_{i o}{ }^{2}-r_{p i}{ }^{2}} .
$$

If $X_{o}$ were omitted from this regression, $Y$ would be regressed on two variables and the regression coefficients would be found by substituting $X_{i}$ for $X_{o}$ in the equations labeled 1 above:

$$
B_{p}=\frac{r_{y p}-r_{y i} r_{p i}}{1-r_{p i}{ }^{2}} \quad B_{i}=\frac{r_{y i}-r_{y p} r_{p i}}{1-r_{p i}{ }^{2}},
$$

The estimate of the effect of the primary predictor $\left(B_{p}\right)$ would be biased to the extent that the correlations between the omitted variable and the other variables are not equal to zero. For example, if the omitted variable and the covariate were not correlated (i.e., $r_{i o}=0$ ), then the coefficient associated with the primary predictor would be

$$
B_{p}=\frac{r_{y p}-r_{y i} r_{p i}-r_{y o} r_{p o}}{1-r_{p i}^{2}-r_{p o}^{2}} .
$$

Therefore, omitting $X_{o}$ from the regression in this instance would bias the estimate of the effect attributed to $X_{p}$ by the full effect of $X_{o}$ on the criterion variable to the extent that $X_{o}$ was correlated with $X_{p}$. However, if the omitted variable and the covariate were correlated (i.e., $r_{i o}<>0$ ), then the bias in the estimate of the effect of the primary predictor caused by omitting $X_{o}$ would be attenuated.

To illustrate this result, the regression coefficients for the primary predictors in the data sets in Table 1 were estimated. The only difference between these data sets is that for data set A the correlation between $X_{i}$ and $X_{o}$ is .1, whereas for data set B that correlation is .7. For data set $\mathrm{A}$, the regression coefficient for $X_{p}$ in a regression of $Y$ on $X_{p}, X_{i}$, and $X_{0}$ is .11. If $X_{o}$ were omitted from this regression, the coefficient for $X_{p}$ again would equal .21. For data set $\mathrm{B}$, the regression coefficient for $X_{p}$ in a regression of $Y$ on $X_{p}, X_{i}$, and $X_{o}$ is .21. If $X_{o}$ were omitted from this regression, the coefficient for $X_{p}$ would equal .21. Clearly, the effect of omitting $X_{o}$ on the regression coefficient of the primary predictor is much greater when the correlation between the omitted variable and the covariates is small.

In sum, the bias in the regression coefficient for a primary predictor caused by the omission of a variable can be determined from the formula for the regression coefficient of the primary predictor that is derived from a model containing the primary predictor, the original covariates, and the omitted variable. This equation is a function of several unknown quantities: the correlation between the omitted variable and the criterion variable $\left(r_{y o}\right)$, the correlation between the omitted variable and the primary predictor $\left(r_{p o}\right)$, and the correlations between the omitted variable and the covariates $\left(r_{i o} s\right)$.

When these correlations are known, they can be used to determine the effect of the omitted variable on the effect attributed to the primary predictor variable. Of course, this is a rare occurrence. If this information had been available, the omitted variable would probably have been included in the original analysis. One can often obtain reasonable estimates of these correlations from other similar samples or archival sources, but in many cases even this information will not be available. In these instances, one can still obtain an estimate of the effect of an omitted variable because these correlations cannot vary freely. Their values are constrained by the limits of mathematical possibility and by the limits of plausibility.

\footnotetext{
${ }^{5}$ The equations for the various parameters and statistics in this section can be found in some form in most texts on multiple regression analysis (e.g, Cohen \& Cohen, 1983; Pedhazur, 1982).
} 
Table 1

Correlations and Regressions Between Three Predictors and a Dependent Variable

\begin{tabular}{|c|c|c|c|c|c|c|c|c|}
\hline Variable & \multicolumn{4}{|c|}{$r_{i 0}=.1$} & \multicolumn{4}{|c|}{$r_{i o}=.7$} \\
\hline \multicolumn{9}{|c|}{ Correlations } \\
\hline Variable & 1 & 2 & 3 & $Y$ & 1 & 2 & 3 & $Y$ \\
\hline 1. $X_{p}$ & - & .4 & .3 & .3 & - & .4 & .3 & .3 \\
\hline 2. $X_{i}$ & .4 & - & .1 & .3 & .4 & - & .7 & .3 \\
\hline 3. $x_{0}$ & .3 & .1 & - & .4 & .3 & .7 & - & .4 \\
\hline
\end{tabular}

Coefficients for regressions on $Y$

\begin{tabular}{lcccccccc} 
& $X_{p}$ & $X_{i}$ & $X_{o}$ & $R^{2}$ & $X_{p}$ & $X_{i}$ & $X_{o}$ & $R^{2}$ \\
Variables in models & & & & & & & & \\
$X_{p}, X_{i}, X_{0}$ & .11 & .22 & .35 & $.24^{\mathrm{a}}$ & .21 & -.04 & .36 & $.20^{\mathrm{b}}$ \\
$X_{p}, X_{i}$ & .21 & .21 & - & $.13^{\mathrm{c}}$ & .21 & .21 & - & $.13^{\mathrm{c}}$ \\
\hline
\end{tabular}

Note. $N=500$.

${ }^{\mathrm{a}} F(3,496)=51.4, p<.001 .{ }^{\mathrm{b}} F(3,496)=40.4, p<.001 .{ }^{\mathrm{c}} F(2$, $497)=36.7, p<.001$.

\section{Limits of Possibility}

The correlation between any two variables is constrained by the relation that each variable has with all other variables. With three variables, $X_{i}, X_{j}, X_{k}$, the correlation between $X_{i}$ and $X_{j}$ must lie within the range defined by:

$$
r_{i k} r_{j k} \pm \sqrt{\left(1-r_{i k}^{2}\right)\left(1-r_{j k}^{2}\right)}
$$

For example, if $r_{i k}=.6$ and $r_{i k}=.7$, then $r_{i j}$ must be greater than -.15 and less than .99 . Values of $r_{i j}$ outside these limits are mathematically impossible. Only when both $r_{i k}$ and $r_{j k}$ equal zero can $r_{i j}$ vary from -1 to +1 . When there are more than three variables, the correlations between all triples of variables must satisfy these constraints. In practice, these constraints can be quite restrictive.

\section{Limits of Plausibility}

In addition to the constraints imposed by the mathematics of correlation matrices, one's knowledge of the variables being studied imposes other constraints. For example, one may not know the correlation between introversion and scores on a test of mathematical ability, but given what is known about personality measures and academic ability tests, it would be completely implausible for this correlation to exceed .5 or .6 . To determine whether the omission of a variable has an important effect on the estimate of a coefficient in a linear model, one can determine what patterns of correlations result in estimates of this coefficient that are substantially different from the estimate originally obtained, and then evaluate whether these patterns of correlations lie within the limits of possibility and plausibility.

What constitutes a "substantial difference" in a regression coefficient depends on the form of the hypothesis being tested. If a precise prediction of the size of the effect is made, then a substantially different coefficient is one that is significantly different from the predicted value. However, predictions of this sort are rare in the social sciences. In most cases, theoretical predictions are limited to the existence and direction of the effects. In these instances, a substantially different coefficient is one that is not significantly different from zero or one that is significant and of opposite sign from the value originally obtained. Therefore, to determine whether a substantially different estimate of the effect of a predictor would have been obtained had an omitted variable been included in a model, one must determine the sign and significance of the regression coefficient for the primary predictor that would be obtained given a pattern of correlations among the criterion, the omitted variable, and the covariates.

There are several ways to calculate the significance of a regression coefficient, all of which yield identical results when the predictors are uncorrelated. When the predictors are correlated, one of the most useful tests of significance is the test of the increment in the proportion of variance in the criterion variable explained by the predictor variable beyond the variance accounted for by the other predictors included in the model. The increment in the proportion of variance explained by a predictor in a three-variable model is

$$
\operatorname{prop}(\mathrm{var})=\frac{B_{p}{ }^{2}}{\left(1-r_{i o}{ }^{2}\right) /\left(1+2 r_{p i} r_{i o} r_{p o}-r_{p o}{ }^{2}-r_{i o}{ }^{2}-r_{p i}{ }^{2}\right)},
$$

where $B_{p}{ }^{2}$ is the square of the standardized regression coefficient for that predictor in a model including the other variables $\left(X_{i}\right.$ and $X_{o}$ ). This quantity can be tested against the residual variance in an $F$ ratio:

$$
F(1, N-k-1)=\frac{\text { prop.(var) }}{\left(1-R^{2}\right) /(N-k-1)},
$$

where $k=$ the number of predictors (including the omitted one), $N$ is the total number of observations, and $R^{2}$ is the proportion of variance in $Y$ explained by the model. When the expression for $B_{p}$ in Equation 2 is substituted for $B_{p}$ in this expression, Equation 3 can be solved for $r_{y o}$ in terms of $F r_{s o}$, and $r_{p g}$. The resulting expression is a quadratic equation whose two roots, when they exist, determine the limits of the range of $r_{y o}$ over which the regression coefficient for the primary predictor is nonsignificant. Because the regression coefficient for a predictor variable is a linear function of $r_{y a}$, the sign of the coefficient can be determined easily for any value of $r_{y o}$ (see Appendix A for a more complete mathematical treatment).

Thus, in the three-variable case (a primary predictor, a covariate, and an omitted variable) for any pattern of correlations between the predictors and the omitted variable (i.e., $r_{p o}$ and $r_{i o}$ ), one can determine the size of the correlation between the omitted variable and the criterion variable $\left(r_{y o}\right)$ that would be required for the omitted variable to reduce the effect attributed to the primary predictor to nonsignificance or to reverse its sign.

Figure 1 illustrates the results of this "omitted variables analysis." For an omitted variable to reduce the effect attributed to the primary predictor to nonsignificance or reverse its sign, it must demonstrate a pattern of correlations that is represented as a point in Figure 1 "outside" (i.e., not between) the two surfaces. All other patterns of correlations are either impossible or 


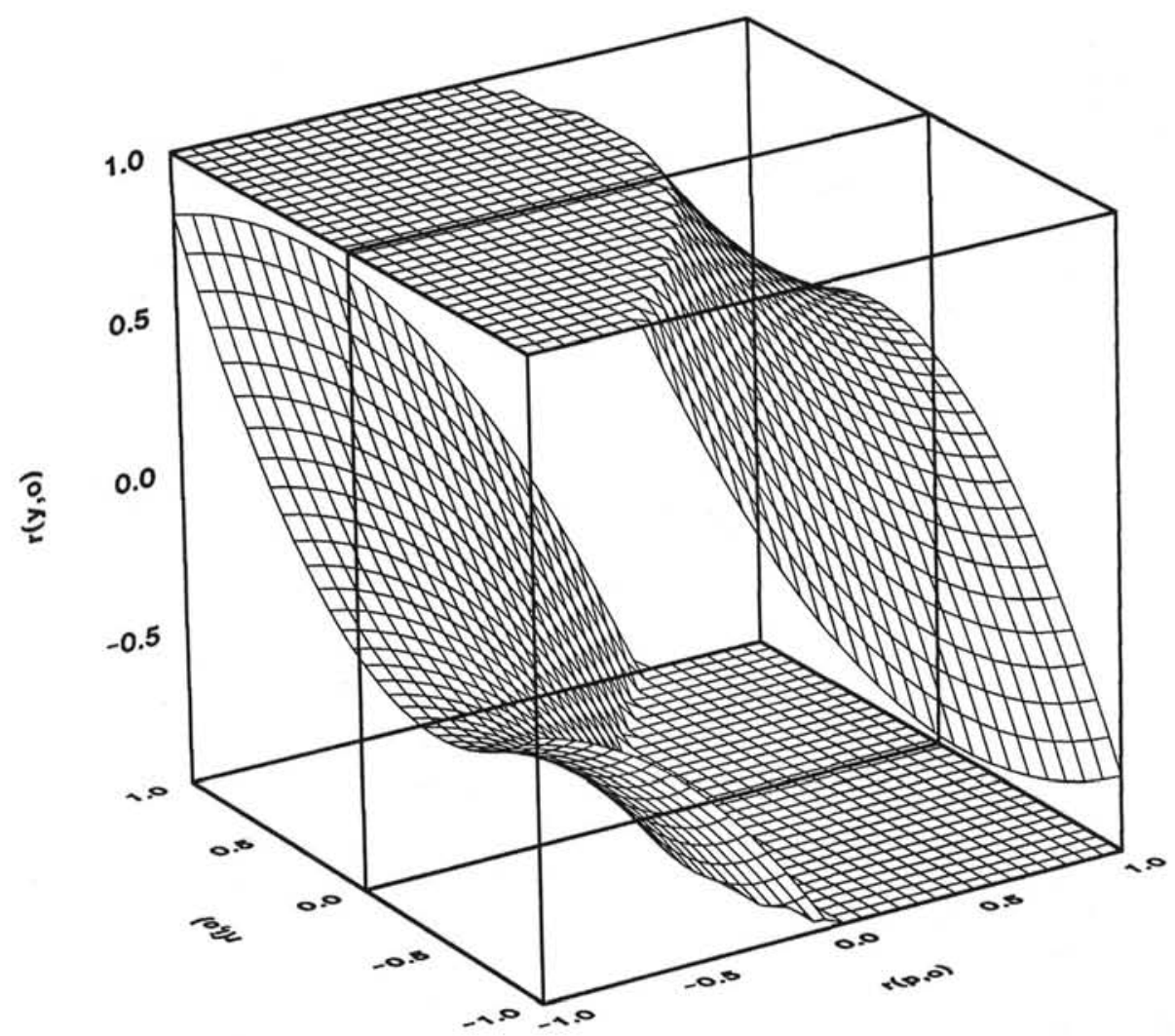

Figure 1. Values of correlations with the omitted variable that would result in substantially different estimates of the effect of the primary predictor. (Patterns of correlations represented by points not between the surfaces would result in substantially different estimates of the effect of the primary predictoror they are mathematically impossible. For clarity, the limits of mathematical possibility have not been drawn on this figure.)

result in significant primary predictor effects of unchanged sign.

Figure 2 is a vertical slice drawn from Figure 1 (marked in Figure 1 by the solid line at $r_{i o}=0$ ). It demonstrates the effect on the coefficient of the primary predictor of different patterns of correlations between the omitted variable and the primary predictor $\left(r_{p o}\right)$ and criterion variable $\left(r_{y o}\right)$ for a given correlation between the covariate and the omitted variable $\left(r_{i o}\right)$. The solid lines in this figure represent the limits of mathematical possibility. The patterns of correlations represented by points lying outside the area defined by these lines (the area shaded with diagonal lines) are impossible. The regression coefficient for the primary predictor is not significant for the patterns of correlations represented as points that lie within the area shaded by horizontal lines. The dashed lines that border this area represent the two roots of the omitted variables equation. The dashed line that lies within the area shaded by horizontal lines represents the values for which the regression coefficient of the primary predictor is equal to zero. On one side of this line, the coefficient takes on positive values; on the other side (the area shaded by vertical lines), it takes on negative values. In this example, the regression coefficient for the primary predictor was positive in a regression without the omitted variable. Therefore, a substantially different coefficient is one that is negative or not significantly different from zero. The shaded areas in Figure 2 represent patterns of correlations that would yield this result or that are impossible. For all other patterns of correlations with the omitted variable (represented in Figure 2 by unshaded areas), the regression coefficient for the primary predictor variable would remain positive and statistically significant. $^{6}$

\footnotetext{
${ }^{6}$ One could develop a summary statistic reflecting how likely it is that an omitted variable could explain the effects attributed to the primary predictor. Those patterns of correlations with the omitted variable for which the estimates of the regression coefficient of the primary predictor are significant and of the same sign as the estimated coefficient describe a volume within the "correlation cube" defined by the correlations between the omitted variable and the primary predictor, other predictors, and criterion. The ratio of this volume to the total volume within the limits of mathematical possibility could provide the desired summary statistic. However, these areas represent patterns of correlations that are not equally likely; therefore, the points within the correlation cube would have to be weighted to reflect their likelihood of occurrence, and it is not clear how these weights should be chosen. In most cases, the obvious functions (c.g., weighting the patterns of correlations by the inverse square of their distance from the origin) probably would not reflect reality.
} 


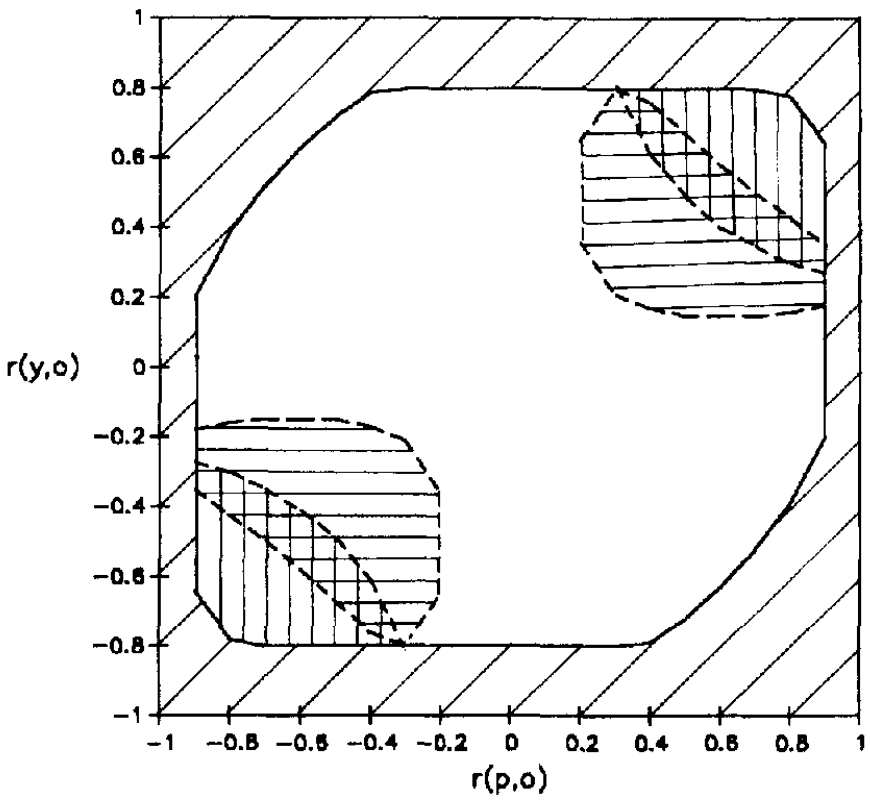

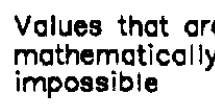

Volues for which primary predictor is significont and of unchonged sign

Values for which primary predictor is non-aignificant

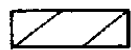

Volues for which primary predictor is significont but of reversed sign

Values for which primary predictor

is non-significant

and of reversed sign

Figure 2. The influence of patterns of correlations with the omitted variable on the estimate of the effect of the primary predictor with the correlation between the omitted variable and the covariate held constant. (This figure corresponds to the slice of Figure 1 marked by the solid line at $r_{10}=0$. The limits of mathematical possibility not shown in Figure 1 are protrayed here.)

\section{A Three-Variable Example}

To illustrate the utility of omitted variables analyses, this technique was applied to a hypothetical study of perceived justice. These data are summarized in Table 2.

There are several theories of perceived justice, each of which stresses different theoretical variables. One approach proposes that for participants in an adjudicative process to believe that that process is just, the participants must believe that the adjudicators are concerned with their welfare (e.g., Cohen, 1986). Another approach suggests that the adjudicators must appear to be fair and unbiased (e.g., Folger, 1977); a third states that it is the importance of the issue being adjudicated that is critical (e.g., Heinz, 1985). According to this approach, individuals will accept an adjudicative process irrespective of the outcome of that process only when the issues being judged are of relatively little importance. Finally, several investigators (cf. Tyler, 1987) have suggested that in an adjudicative process, the more partici-

Table 2

Correlations Between Variables in a Perceived Justice Study

\begin{tabular}{lcccc}
\hline & \multicolumn{5}{c}{ Variable } \\
\cline { 2 - 6 } \multicolumn{1}{c}{ Variable } & 1 & 2 & 3 & 4 \\
\hline 1. Perceived justice & - & & & \\
2. Perceived concern & .459 & - & & \\
3. Perceived fairness & .561 & .369 & - & \\
4. Outcome importance & .494 & .007 & .148 & -190 \\
5. Expression allowed & .504 & -.004 & .154 & .190 \\
\hline
\end{tabular}

Note. $N=240$. pants feel that they have been allowed to express their points of view, the more just they will perceive the process.

A researcher interested in the effect of the perceived fairness of the adjudicator on the perceived justice of an adjudicative process might regress a measure of the participants' beliefs in the fairness of the adjudicator on their perceptions of the justice of the process while "controlling" for differences in perceptions of the adjudicator's concern for the welfare of the participants. On the basis of the data summarized in Table 2, this regression yields the model

$$
\begin{aligned}
& \text { Perceived Justice }=.454 \times \text { Perceived Fairness } \\
& +.291 \times \text { Concern about Welfare } \\
& \qquad \begin{array}{l}
R^{2}=.388, \quad F(2,238)=75.51, \quad p<.001 .
\end{array}
\end{aligned}
$$

Both variables have significant effects at $p \leq .001$. Another researcher could question these results because no measure of the degree to which the participants' feel that they have been allowed to express their points of view was included, and there is reason to believe that the perceived justice of an adjudicative process is affected by this factor. To judge whether the omission of this variable seriously biased the effects attributed to perceived fairness, an omitted variables analysis was performed on these data using a computer program written by the author. ${ }^{7}$ Table 3 reports the results of this analysis.

The rows in Table 3 are defined by correlations of the omitted variable with perceived concern about the participants' welfare. The columns are defined by correlations of the omitted

\footnotetext{
${ }^{7}$ OMITVAR (1988) by R. Mauro, written in True Basic ${ }^{\$}$.
} 
ROBERT MAURO

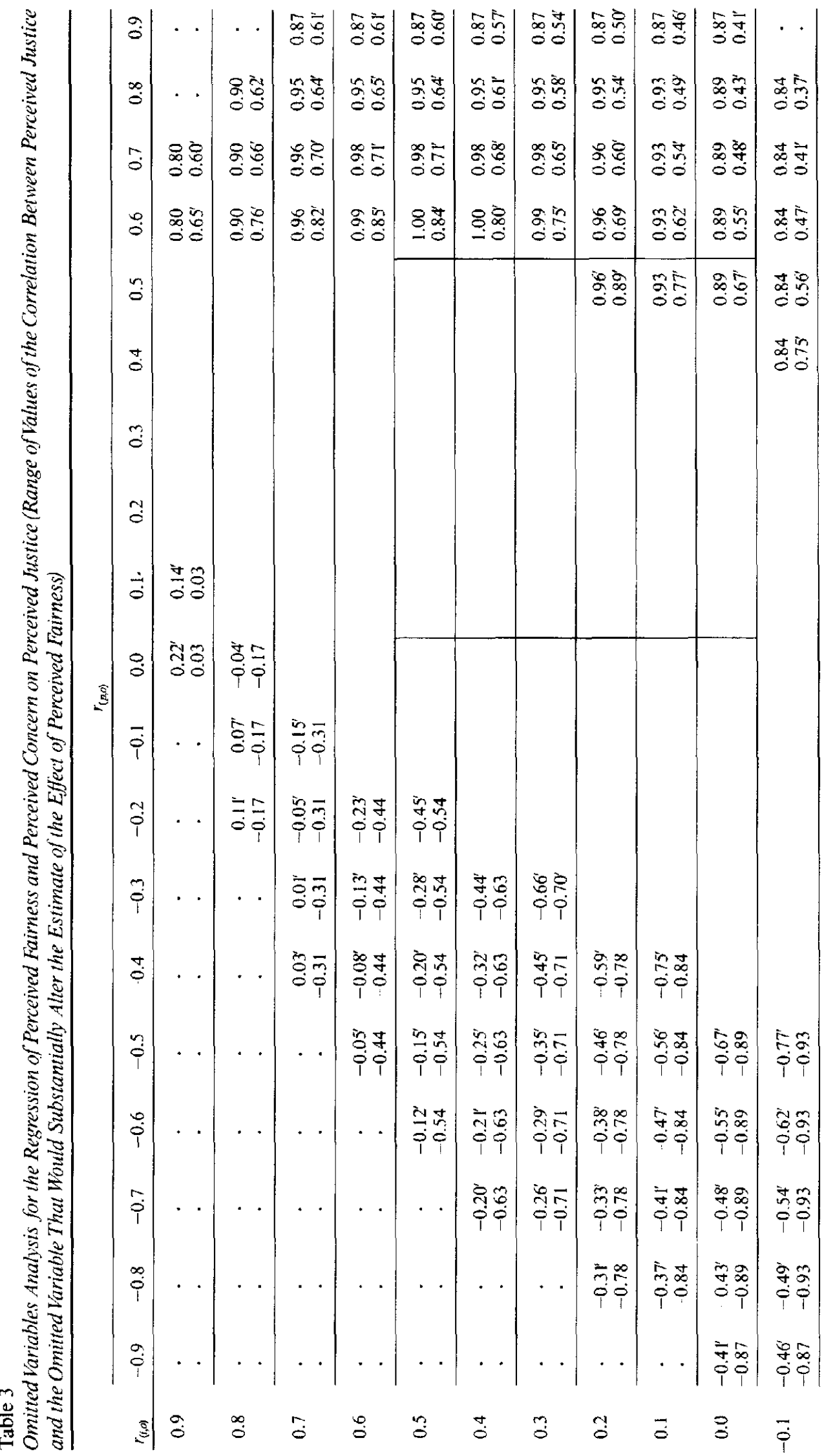




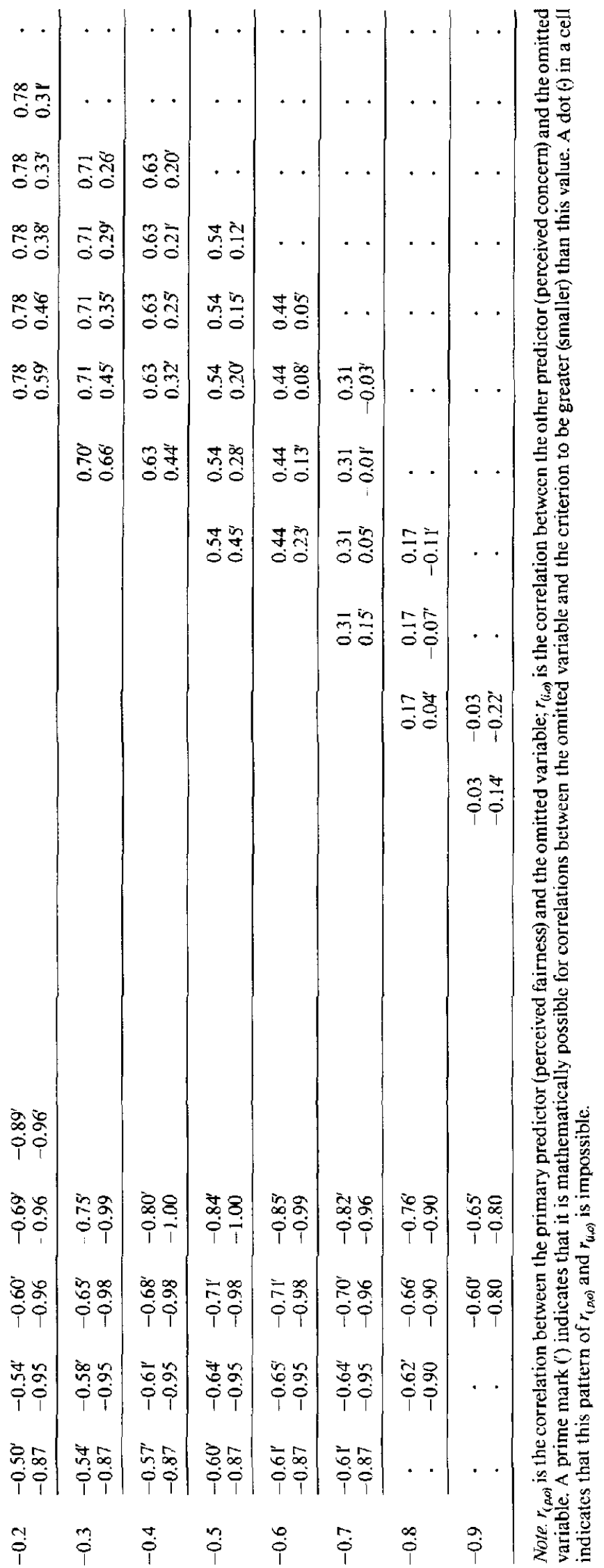

variable with perceived fairness. Within each cell of the table are two numbers that define a range of correlations between the omitted variable and perceptions of the justice of the process. If the omitted variable has a correlation with the perceived justice variable that falls within this range, then that omitted variable could account for the effects attributed to fairness-given the correlations with perceived fairness and concern about welfare that define that cell of the table. An empty cell indicates that given the pattern of correlations that define that cell, the ormitted variable cannot account for the effects attributed to perceived fairness, no matter what the correlation between the omitted variable and perceived justice. A dot in a cell indicates that that pattern of correlations between perceived fairness, concern about welfare, and the omitted variable is impossible.

If one were to assume that the correlations between the participants' feelings about the amount of expression that they were allowed (the omitted variable) and their perceptions of the adjudicator's fairness and concern for their welfare are both positive and less than .5, then the relevant portion of Table 3 is enclosed in the rectangle. Over most of this range, an omitted variable could not account for the effect attributed to fairness. When it is possible, the omitted variable would have to correlate more than .67 with perceived justice for it to account for the effect attributed to perceived fairness.

In fact, data on "expression allowed" are available for this sample (see Table 2). Given the obtained pattern of correlations, feeling about the amount of expression allowed could not account for the effects attributed to fairness. Indeed, when a variable measuring the participants' feelings about the amount of expression allowed is added to the model containing perceived concern for welfare and perceived fairness, the effect of fairness remains significant:

Perceived Justice $=.373 \times$ Perceived Fairness +.323

$$
\times \text { Concern about Welfare }+.448 \times \text { Expression Allowed }
$$

$$
R^{2}=.583, \quad F(3,237)=100.42, \quad p<.001 .
$$

\section{Multiple Predictors}

This technique for analyzing the effects of an omitted variable can be extended easily to models that contain more than two predictors and an omitted variable (see Appendix A). Each additional covariate simply adds a dimension to the table. However, for models with a large number of covariates this method may become unwieldy. In these cases, one can rely on an approximate method. To do so, the covariates are combined into a single variable that is a linear combination of the original covariates optimally weighted to predict the criterion. This new variable is then used as the covariate in the omitted variables analysis already described.

The desired linear combination can be obtained by regressing the criterion on the covariates. The coefficients from this regression are the weights needed to construct the linear combination. The multiple correlation obtained (as the square root of the $R^{2}$ value) can then be used as the correlation between the criterion and the covariate in the three-variable omitted variables procedure. The correlations between the criterion and the 
omitted variable and primary predictor can be estimated by applying the standard score equation (see Appendix B):

$$
r\left(\sum B_{i} x_{i}, x_{v}\right)=\frac{\sum_{i=1}^{k} B_{i} r\left(x_{o}, x_{i}\right)}{\sum_{i=1}^{k} B_{i}^{2}+\sum \sum_{i \ll j} B_{i} B_{j} r\left(x_{i}, x_{j}\right)},
$$

where $k=$ the number of covariates, $x_{v}$ represents either the primary predictor or the omitted variable, and the $B_{i}$ are the standardized regression coefficicnts for the covariates $\left(X_{i}\right)$ from the regression of these predictors on the criterion. If one is able to specify the limits of plausibility for the correlations between each of the covariates and the omitted variable, one can determine the limits of plausibility for the correlation between the linear combination of covariates and the omitted variable.

The correlation between a variable and a linear combination of variables given by Equation 4 cannot represent all of the information contained in the correlations between the original variables. All of this information is needed to estimate the effects of the predictors in a regression analysis. The results of an omitted variables analysis based on a linear combination of covariates can only approximate the results that would be obtained by applying the omitted variables analysis to the original covariates. Depending on the pattern of correlations among the omitted variable, the primary predictor, and the covariates, the effect of the primary predictor may be under-or overestimated (see Appendix C).

However, a consistently conservative estimate of the effect of the primary predictor can be obtained by substituting the multiple correlation of the primary predictor with the covariates for the correlation between the primary predictor and the linear combination of covariates. This correlation can be obtained as the square root of the $R^{2}$ value from the regression of the covariates on the primary predictor. It represents the correlation between the primary predictor and a linear combination of covariates optimally weighted to predict the primary predictor (instead of the criterion). This substitution ensures that the value used for the correlation between the covariate and the primary predictor will not underestimate the variance shared by the primary predictor and the covariates. This number will generally be higher than the correlation between the primary predictor and the linear combination of covariates optimally weighted to predict the criterion. Using this value will result in a consistent underestimation of the ranges of correlations between the omitted variable and the criterion variable that can be achieved without reducing the estimate of the effect of the primary predictor to nonsignificance. This will work against the hypothesis that the primary predictor has a significant effect on the criterion variable.

\section{A Multiple-Variable Example}

The data in Table 2 were used again to illustrate the use of these approximations in an omitted variables analysis. If one regresses perceived fairness, perceived concern, and importance of the issue on perceived justice, the result is model A2 in Table 4. Once again, all of the predictors have highly significant effects $(p<.001)$ on perceived justice. However, a measure of the participants' feelings about the amount of expression allowed has again been omitted from this analysis.

Table 4

Regression Coefficients and Summary Statistics for Regressions on Perceived Justice

\begin{tabular}{|c|c|c|c|c|c|c|}
\hline \multicolumn{7}{|c|}{ Models with original predictors only } \\
\hline & Fairness & Concern & Importance & Expression & $R^{2}$ & Change in $R^{2}$ \\
\hline $\mathrm{A} 1$ & - & .456 & .491 & - & .452 & \\
\hline A2 & .380 & .316 & .436 & - & .573 & .121 \\
\hline A3 & - & .458 & .409 & .428 & .628 & \\
\hline A4 & .322 & .339 & .371 & .385 & .714 & .086 \\
\hline \multicolumn{7}{|c|}{ Models with linear combination without adjustment } \\
\hline & Fairness & Linea & bination & Expression & $R^{2}$ & Change in $R^{2}$ \\
\hline B1 & - & & & - & .452 & \\
\hline $\mathrm{B} 2$ & .368 & & & - & .569 & .117 \\
\hline B3 & - & & & .420 & .625 & \\
\hline B4 & .321 & & & .386 & .714 & .089 \\
\hline \multicolumn{7}{|c|}{ Models with linear combination-conservative approximation } \\
\hline & Fairness & Linea & nbination & Expression & $R^{2}$ & Change in $R^{2}$ \\
\hline $\mathrm{C} 1$ & - & & & - & .452 & \\
\hline $\mathrm{C} 2$ & .349 & & & - & .554 & .102 \\
\hline C3 & - & & & .420 & .625 & \\
\hline $\mathrm{C} 4$ & .303 & & & .390 & .701 & .076 \\
\hline
\end{tabular}

Note. $N=240$. All figures significant at $p<.001$. Dashes for coefficient for a predictor indicate that variable was not in the model. 
An omitted variables analysis can be performed on these data using the approximate method described above. To do so, one must first regress the criterion variable on the covariates to obtain the weighted linear combination of predictors and the correlation between this new variable and the criterion (the square root of the $R^{2}$ value from this regression). Then, one can either calculate the correlation between perceived fairness and this new variable or use the conservative procedure and regress the covariates on the primary predictor to obtain the multiple correlation that will be used as the correlation between the new covariate and the primary predictor. The results reported in Table 5 were obtained using the first procedure.

If one assumes that the correlation between the omitted variable ("expression allowed") and perceived concern and perceived importance are positive and less than .5 , then the correlation between the linear combination of covariates and the omitted variable can be calculated by Equation 4 to be between 0 and .7. If one also assumes that the correlation between the omitted variable and perceived fairness is between 0 and .5 (as in the three-variable example), then the relevant part of Table 5 is the portion enclosed in the rectangle. Over most of this range, it is impossible for the omitted variable to account for the effects attributed to perceived fairness. When it is possible for the omitted variable to account for the effects attributed to perceived fairness $\left(r_{p o}=.5,0 \leq r_{i o} \leq .2\right)$, the correlation between the omitted variable and perceived justice must be greater than .54

Data on "expression allowed" is available for this sample (see Table 2). When this variable is added to a model containing perceived fairness, concern, and importance, the effect attributed to perceived fairness remains positive and significant ( $\mathrm{cf}$. model A4 in Table 4).

The effect of using the approximate methods on these data instead of the full correlation matrix can be seen in Table 4 . This table is divided into three sections. In the top section the original variables are used. The proportion of variance in the criterion explained by the primary predictor is calculated for two models-one with the two covariates and one with these two covariates and the previously omitted variable. In the middle section (labeled "linear combination without adjustment"), the effect of the primary predictor is estimated from models in which the covariates have been replaced by a linear combination of those covariates optimally weighted to predict the criterion. In the bottom section, the "conservative" approach is used. The effect of the primary predictor is calculated from correlation matrices in which the correlation between the primary predictor and the linear combination has been replaced by the multiple correlation between the primary predictor and the covariates.

When "expression allowed" is omitted from the models, all three estimates of the effect of the primary predictor are similar. The proportion of the variance in the criterion attributed to the primary predictor is largest in the model (A2) based on the original variables and smallest in the model $(\mathrm{C} 2)$ incorporating the multiple correlation between the primary predictor and the covariates. When the omitted variable is included in the models, all of the estimates of the effect of the primary predictor are again similar. The proportion of variance in the criterion attrib- uted to the primary predictor is again smallest in the model (C4) incorporating the multiple correlation between the primary predictor and the covariates. Although the difference is small, the effect attributed to the primary predictor is larger in the model (B4) using the linear combination without adjustment than it is in the model (A4) based on the original variables.

\section{An Application: Racial Discrimination in Capital Sentencing}

Of course, an omitted variables analysis is useful primarily when a potentially important variable actually has not been measured. An example (with important consequences) of this use of omitted variables analysis is its application to the study of racial discrimination in capital sentencing. In 1972, the U.S. Supreme Court declared that all death penalty laws then in effect were unconstitutional and vacated all death sentences then in effect (Furman v Georgia, 1972). The reason for this decision (as best as it can be deciphered) was that the death penalty under these laws was "arbitrary" and "discriminatory" In response to this ruling, new death penalty statutes were enacted in many states, and in 1976 the Supreme Court upheld many of these laws because they appeared to the Court to avoid the pitfalls that led to discrimination under the earlier statutes. By the early 1980s it became clear that racial disparities in capital sentencing were still present, and challenges to these statutes were mounted (cf. Gross \& Mauro, 1988).

However, evidence of a racial disparity does not demonstrate the existence of racial discrimination. In most cases, the legal proof of racial discrimination requires evidence of racial disparities that cannot be attributed to the operation of legally permissible factors. To determine whether there was racial discrimination in capital sentencing, Gross and Mauro (1984) analyzed homicide data for 5 years from eight states: Arkansas, Florida, Georgia, Illinois, Mississippi, North Carolina, Oklahoma, and Virginia. In every state studied, killers of Whites were much more likely to receive the death penalty than were killers of Blacks--even when the joint effects of many other variables are considered (the presence of other contemporaneous felonies, whether muitiple victims were killed, the sex of the victim(s), the relationship of the victim(s) to the defendant, whether a firearm was used, and the race of the defendant).

Unfortunately, information on some variables that affect capital sentencing decisions could not be obtained. Chief among these is the seriousness of the prior criminal record of the defendant. At the time, Gross and Mauro (1984) argued that this omitted variable could not explain the effect attributed to the race of the victim because to do so it would have to be highly correlated with the race of the victim and not highly correlated with the other variables included in the analyses (such as the presence of contemporaneous felony circumstances and the race of the defendant). This issue can be made more precise by applying an omitted variables analysis to these data.

Table 6 displays the results of an omitted variables analysis using the conservative approximate method applied to the data 


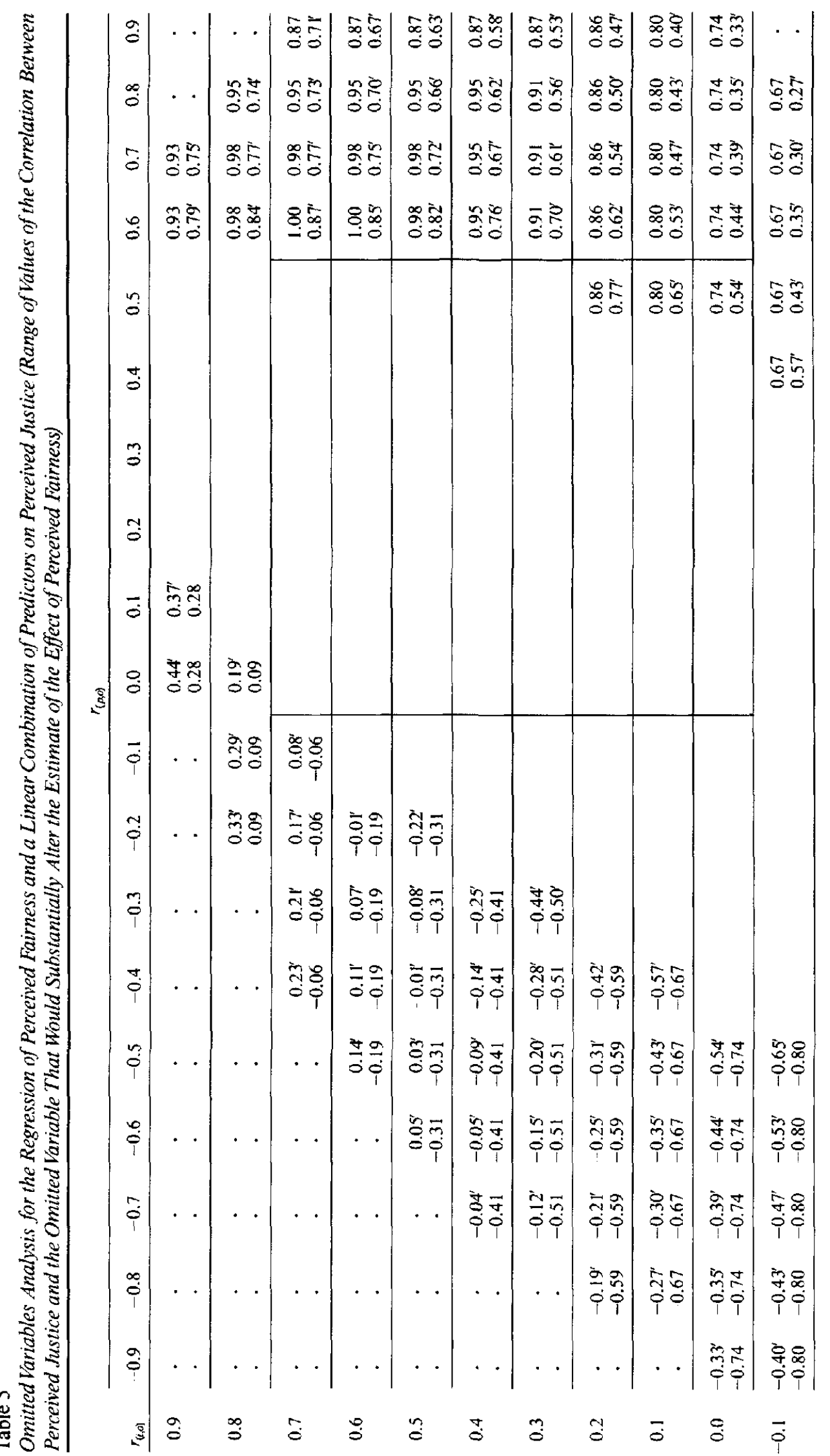




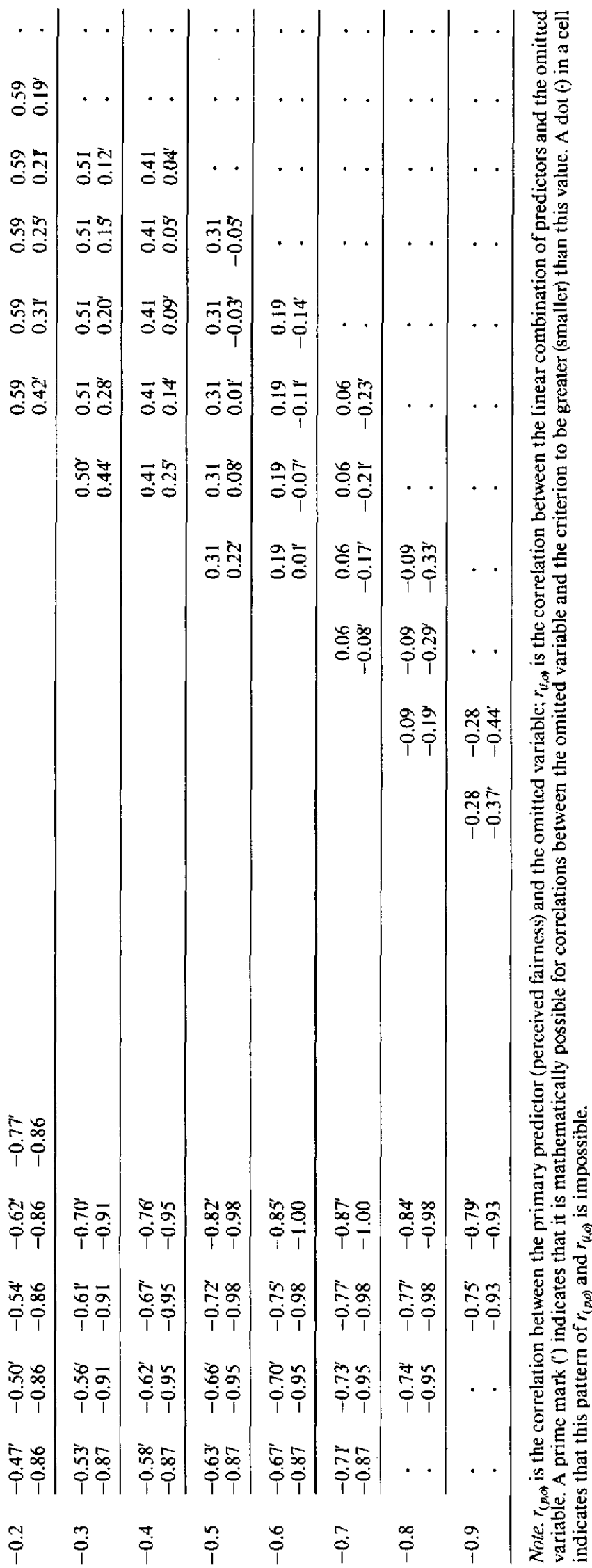

from Georgia. ${ }^{8}$ On the basis of other studies of capital sentencing in similar populations, the correlation between the victim's race and the seriousness of the defendant's prior criminal record can be estimated to be nearly zero (this correlation is .03 in Baldus, Woodworth, \& Pulaski 's, 1987, extensive study of a sample of 2,176 cases from Georgia). It is clear from Table 6 that given this correlation, there is no pattern of correlations between the defendant's record and the other included variables and the probability of receiving the death penalty that would cause the effect of victim's race to become nonsignificant. Even if the actual correlation between the defendant's prior record and the victim's race were 6 times greater than our estimate, it would still be impossible for the defendant's criminal record to account for the effect attributed to the victim's race. ${ }^{9}$

\section{Conclusion}

Omitted variables analyses are best viewed as techniques for evaluating the probable validity of the hypothesis that an omitted variable can account for the effect attributed to a predictor included in an analysis when the information needed for a proper test is unavailable. Omitted variables analyses can only help illuminate the problems of misspecification due to an omitted variable; they are no substitute for additional information. Regression analyses can suffer from several possible shortcomings in addition to left out variables error. There may be other problems with the specification of the model (e.g., inclusion of irrelevant variables and misspecification of the functional form) and problems with the data (e.g., errors in measurement, multicollinearity, heteroscedasticity, and autocorrelation). The utility of omitted variables analyses depends on the reliability of the information upon which they are based. An

\footnotetext{
${ }^{8}$ Applying an omitted variables analysis to these data constituted a multistage process. The regressions originally performed on these data used a maximum likelihood technique for fitting a logistic curve rather than the ordinary least squares techniques used by the omitted variables procedure. To use the omitted variables procedure, the model derived by the maximum likelihood logistic regression was reestimated using least squares. The resulting estimated proportions correlated .926 with those obtained from the maximum likelihood regression. This model contained not only victim's race but several interactions of legally permissible variables with victim's race (cf. Gross \& Mauro, 1984). Therefore, two new variables were created: one that was an optimally weighted combination of victim's race and its interactions, and another that was an optimally weighted combination of the legally permissible variables (and their interactions). The new victim's race variable was then regressed on the new legally permissible factors variable and the resulting residualized victim's race variable to obtain the multiple correlation used in the analyses reported here. The correlation between the victim's race variable and the criterion was .261 . The correlation between the combination of legal variables and the criterion was .512 .

${ }^{9}$ The Supreme Court has now more or less acknowledged that race continues to play a major role in capital sentencing in the United States (McCleskey v. Kemp, 1987), but the Court has decided to do nothing about this form of discrimination and to refuse to hear future claims based on it.
} 
ROBERT MAURO

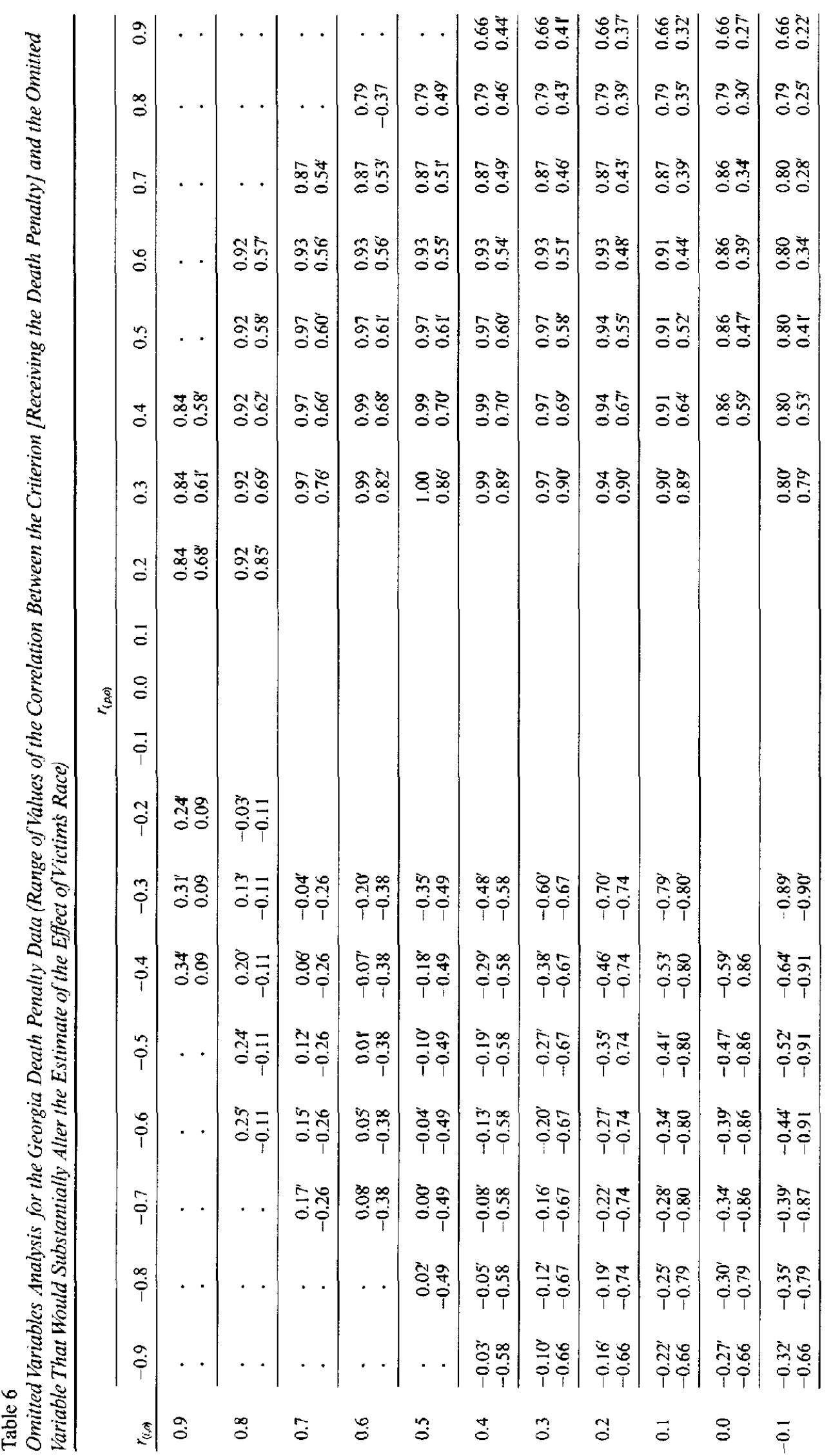




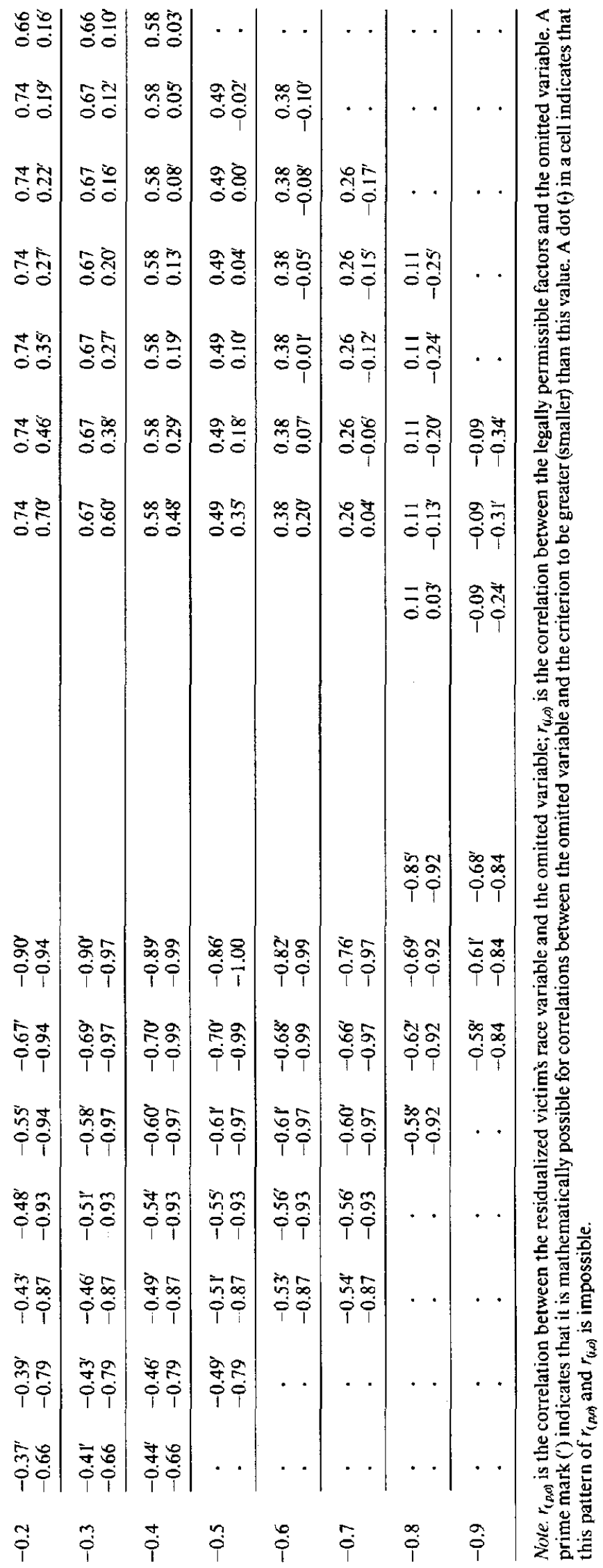

omitted variables analysis cannot correct for poor sample selection. If the relations between the variables in the sample do not reflect the relations in the population to which one would like to generalize, the results of the omitted variables analysis will not generalize either. If the correlations used are unreliable, the error in these estimates will be carried over into the results of the omitted variables analysis.

For an omitted variable to account for the effects attributed to a predictor included in an analysis, it must demonstrate one of a set of patterns of relations with that predictor, the criterion variable, and the other predictors in the analysis. To criticize a finding because a variable was omitted from the analysis is to hypothesize that one of these patterns of relations does, in fact, exist. Omitted variables analysis can be used to add precision to this hypothesis by specifying the parameters of the hypothesis and by providing a framework for its evaluation.

\section{References}

Baldus, D., Woodworth, G., \& Pulaski, C. (1987). Equal justice and the death penalty: $A$ legal and empirical analysis. Unpublished manuscript.

Cohen, J., \& Cohen, P. (1983). Applied multiple regression/correlation analysis for the behavioral sciences. Hillsdale, NJ: Erlbaum.

Cohen, R. L. (1986). Power and justice in intergroup relations. In H. W. Bierhoff, R. L. Cohen, \& J. Greenberg (Eds.), Justice in social relations (pp. 65-86). New York: Plenum Press.

Folger, R. (1977). Distributive and procedural justice: Combined impact of "voice" and peer opinions on responses to inequity. Journal of Personality and Social Psychology. 35, 108-119.

Furman v. Georgia, 408 U.S. 238 (1972).

Gross, S., \& Mauro, R. (1984). Patterns of death. Stanford Law Review, 37, 27-153.

Gross, S., \& Mauro, R. (1988). Death \& discrimination: Racial biases in capital sentencing. Boston: Northeastern University Press.

Heinz, A. (1985). Procedure versus consequence: Experimental evidence of preferences for procedural and distributive justice. In $\mathrm{S}$. Talarico (Ed), Courts \& criminal justice: Emerging issues (pp. 1334). Beverly Hills, CA: Sage.

Kennedy, P. (1985). A guide to econometrics. Cambridge, MA: MIT Press.

Klepper, S., \& Leamer, E. (1984). Consistent sets of estimates for regressions with errors in all variables. Econometrica, 52, 163-183.

Leamer, E. (1978). Specification searches: Ad hoc inference with nonexperimental data. New York: Wiley.

Mariano, R., \& Ramage, J. (1983). Specification error analysis in linear simultaneous systems. In R. Basmann \& G. Rhodes (Eds.), $A d$ vances in econometrics: Volume 2 (pp. 51-95). Greenwich, CT: JAI Press.

McCleskey v. Kemp, 107 S.Ct. 1756 (1987).

Meehl, P. (1971). High school yearbooks: A reply to Schwarz. Journal of Abnormal Psychology, 77, 143-148.

Mood, A., Graybill, F., \& Boes, D. (1974). Introduction to the theory of statistics. San Francisco: McGraw-Hill.

Pedhazur, E. (1982). Multiple regression in behavioral research: Explanation and prediction (2nd ed.). San Francisco: Holt, Rinehart, \& Winston.

Ramsey, J. (1969). Tests for specification errors in classical linear least-squares regression analysis. Journal of the Royal Statistical Society, Series B, 2, 350-371.

Ramsey, J. (1974). Classical model selection through specification 
error tests. In P. Zarembka (Ed.), Frontiers in econometrics (pp. $13-$ 47). New York: Academic Press.

Ramsey, J., \& Schmidt, P. (1976). Some further results on the use of OLS and BLUS residuals in specification error tests. Journal of the American Statistical Association, 71, 389-390.

Reichardt. C., \& Gollob, H. (1986). Satisfying the constraints of causal modeling. In W. Trochim (Ed.), Advances in quasi-experimental de sign and analysis: New directions for program evaluation. No. 31 (pp. 91-107). San Francisco: Jossey-Bass.

Reichardt, C., \& Gollob, H. (1987, October). Setting limits on the bias due to omitted variables. Paper presented at the meeting of the Society of Multivariate Experimental Psychology, Denver, CO.

Rosenbaum, P. (1984). From association to causation in observational studies: The role of tests of strongly ignorable treatment assignment. Journal of the American Statistical Association, 79, 41-48.
Rosenbaum, P. (1986). Dropping out of high school in the United States: An observational study. Journal of Educational Statistics, 11 , 207-224.

Rosenbaum, P., \& Rubin, D. (1983). Assessing sensitivity to an unobserved binary covariate in an observational study with binary outcome. Journal of the Royal Statistical Society, Series B, 45, 212-218.

Rubin, D. (1973). The use of matched sampling and regression adjustment to remove bias in observational studies. Biometrics, $29,185-$ 203.

Thursby, J., \& Schmidt, P. (1977). Some properties of tests for specification error in a linear regression model. Journal of the American Statistical Association, 72, 635-641.

Tyler, T. (1987). Conditions leading to value-expressive effects in judgments of procedural justice: A test of four models. Journal of Personality and Social Psychology, 52, 333-344.

\section{Appendix A}

Using matrix notation, the equation for the regression on a set of $k$ standardized predictors ( $\mathbf{X}$ ) of a standardized criterion ( $\mathbf{Y}$ ) is $\mathbf{Y}=\mathbf{B X}$ The vector of standardized regression coefficients $\mathbf{B}$ is given by $\mathbf{B}=$ $\mathbf{R}^{-1} \mathbf{r}_{y}$, where $\mathbf{R}^{-1}$ represents the inverse of the correlation matrix of predictors and $\mathbf{r}_{y}$ is a vector of correlations between the predictors and the criterion. The coefficient for the $i$ th predictor is therefore

$$
B_{i}=\sum_{i=1}^{k} r_{i j}{ }^{-1} r_{y j}
$$

where $r_{i j}^{-1}$ represents the $i, j$ element of $\mathbf{R}^{-1}$ and $r_{y j}$ represents the $j$ th element of $r_{y}$. The variance explained by this regression $\left(R^{2}\right)$ is given by

$$
\begin{aligned}
R^{2} & =\mathbf{B}^{\prime} \mathbf{r}_{y} \\
& =\left(\mathbf{R}^{-1} \mathbf{r}_{y}\right)^{\prime} \mathbf{r}_{y} \\
& =\mathbf{r}_{y}{ }^{\prime} \mathbf{R}^{-1} \mathbf{r}_{y} \\
& =\sum_{i=1}^{k}\left(r_{y i}\left(\sum_{j=1}^{k} r_{y j} r_{i j}{ }^{-1}\right)\right)
\end{aligned}
$$

(where' indicates the transpose of the preceding matrix). The proportion of the variance in the criterion explained by a predictor beyond that accounted for by the other predictors is given by

$$
\frac{B_{i}^{2}}{r_{i i}^{-1}}
$$

where $B_{i}{ }^{2}$ is the square of the regression coefficient of the $i$ th predictor in a model including all of the other predictors. This quantity can be tested in an $F$ ratio:

$$
F(1, N-k-1)=\frac{B_{i}^{2} / r_{i i}^{-1}}{\left(1-R^{2}\right) /(N-k-1)},
$$

where $N$ is the total number of observations.

The effect that an omitted variable $\left(X_{o}\right)$ has on the coefficient of a variable included in a regression model $\left(X_{i}\right)$ is a function of the correlations between the omitted variable and the criterion and the predictors in the model. For any given sample, the above equation can be solved for any one of these correlations in terms of the others. For instance, for any given sample and values of the $r_{i o} \mathrm{~s}$, the values of $r_{y o}$ that would cause the coefficient of the $i$ th variable to be nonsignificant can be found as the two roots of a quadratic equation:

$$
\frac{F\left(r_{i i}^{-1}\right)}{(N-k-1)}=\frac{B_{i}^{2}}{\left(1-R^{2}\right)}
$$

where $F$ represents the value of the $F$ ratio above. For convenience, let the expression on the left-hand side of this equation be represented by $Q$; then this equation can be rewritten as:

$$
\begin{aligned}
& Q=\frac{B_{i}^{2}}{\left(1-R^{2}\right)} \\
& 0=B_{i}^{2}+Q R^{2}-Q \\
& 0=\left(\sum_{j=1}^{k} r_{i j}^{-1} r_{y j}\right)^{2}+Q \sum_{i=1}^{k}\left(r_{y i}\left(\sum_{j=1}^{k} r_{y j} r_{i j}{ }^{-1}\right)\right)-Q
\end{aligned}
$$

Now, assume that the omitted variable is the first variable in the regression model (because the order of the variables in a model is immaterial, this assumption can be made without loss of generality). The right-hand side of the above equation can then be reexpressed in terms of the correlation between the first predictor and the criterion, $r_{y \mathrm{i}}$ :

$$
\begin{aligned}
0= & {\left[\left(r_{i 1}{ }^{-1}\right)^{2}+2 r_{i 1}{ }^{-1} r_{y 1}\left(\sum_{j=2}^{k} r_{i j} r_{y k}\right)+\left(\sum_{j=2}^{k} r_{i j}^{-1} r_{y j}\right)^{2}\right]+Q\left[r_{11}{ }^{-1} r_{y 1}{ }^{2}\right.} \\
& \left.+\left(2 \sum_{j=2}^{k} r_{i j}^{-1} r_{y j}\right) r_{y 1}+\sum_{j=2}^{k} r_{y i}\left(\sum_{j=2}^{k} r_{y j} r_{i j}{ }^{-1}\right)\right]-Q \\
= & {\left[\left(r_{i 1}{ }^{-1}\right)^{2}+Q r_{11}{ }^{-1}\right] r_{y 1}{ }^{2}+\left[2 r_{i 1}{ }^{-1}\left(\sum_{j=2}^{k} r_{i j} r_{y k}\right)+2 Q \sum_{j=2}^{k} r_{i j}^{-1} r_{y j}\right] r_{y 1} } \\
& +\left[\left(\sum_{j=2}^{k} r_{i j}{ }^{-1} r_{y j}\right) 2+Q \sum_{j=2}^{k} r_{y i}\left(\sum_{j=2}^{k} r_{y j} r_{i j}{ }^{-1}\right)-Q\right],
\end{aligned}
$$

which has the form $0=a r_{y 1}{ }^{2}+b r_{y 1}+c$ and has roots given by the familiar quadratic formula

$$
r_{y 1}=\frac{-b \pm \sqrt{b^{2}-4 a c}}{2 a}
$$




\section{Appendix B}

The correlation between a variable $X_{0}$ and a linear combination of random variables $\left(\Sigma a_{i} X_{i}\right)$ is

$$
r\left(X_{o}, \sum a_{i} X_{i}\right) \quad \frac{\sum a_{i} \operatorname{cov}\left(X_{o}, X_{i}\right)}{\sqrt{\left[\operatorname{var}\left(X_{o}\right)\right]\left(\sum_{i=1}^{k} a_{i}^{2} \operatorname{var}\left(X_{i}\right)+\sum \sum_{i<>j} a_{i} a_{j} \operatorname{cov}\left(X_{i} X_{j}\right)\right)}} ;
$$

however, because these variables have been standardized, their variances are equal to 1 , and therefore this equation reduces to the equation in the text (cf. Mood, Graybill, \& Boes, 1974).

\section{Appendix C}

When the assumptions of ordinary least squares regression are met, it will provide estimates of the model parameters that are as good or better than other procedures. The variance explained by a model in which a linear combination of a subset of the predictors has been substituted for those predictors will not exceed the variance explained by a model containing the original predictors. That is,

$$
R_{Y, 1,2 \ldots i, j, \ldots k^{2}} \geq R_{Y, 1,2 \ldots, L^{2}},
$$

where $R_{Y \cdot 1, \ldots k^{2}}$ represents the variance in $Y$ explained by variables 1 through $k$, and $L$ represents the linear combination of variables $j$ through $k$.

If the linear combination is obtained by a regression of the criterion on the subset of predictors, the variance explained by the linear combination will equal that explained by the predictors of which it is composed; that is,

$$
R_{Y, j, \ldots k}{ }^{2}=R_{Y \cdot L}^{2}
$$

Therefore, in a model in which this linear combination has been substituted for a set of predictors $(j \ldots k)$, the effect attributed to the other predictors $(1$...i) will not exceed the effect attributed to those predictors in a model in which the original variables are used.

$$
R_{Y \cdot 1,2 \ldots j \ldots k^{2}}-R_{Y \cdot j, \ldots k^{2}} \geq R_{Y \cdot 1,2 \ldots L^{2}}-R_{Y \cdot L}{ }^{2}
$$

When there is only one predictor not included in the linear combination, the proportion of the variance in the criterion explained by this predictor in the model with the original variables will be greater than or equal to the proportion of variance explained by this predictor in the model with the linear combination.

$$
\begin{gathered}
R_{Y \cdot 1, j \ldots k^{2}}-R_{Y \cdot j, \ldots k}{ }^{2} \geq R_{Y \cdot 1, L}{ }^{2}-R_{Y \cdot L}{ }^{2} \\
R_{Y(1, \ldots k)}{ }^{2}+R_{Y \cdot j, \ldots k}{ }^{2}-R_{Y \cdot j \ldots k}{ }^{2} \geq R_{Y(1, L)}{ }^{2}+R_{Y \cdot L}{ }^{2}-R_{Y \cdot L}{ }^{2} \\
R_{Y(1, j \ldots k)} \geq R_{Y(1, L)}{ }^{2}
\end{gathered}
$$

$\left(R_{Y(1, \ldots, k)^{2}}\right.$ represents the proportion of variance in $Y$ explained by variable $l$ in a model with variables 1 and $j$ through $k$ ). However, when there is more than one predictor not included in the linear combination, this inequality holds only for these variables considered as a set. In the model containing the linear combination, the variance explained by these predictors together will not exceed the variance explained by these variables in the model containing the original variables.

$$
R_{Y(1,2 \ldots i+\ldots k)^{2}} \geq R_{Y(1,2 \ldots i b)}{ }^{2}
$$

The proportion of variance in the criterion explained by any single predictor may be larger in the model with the linear combination.

Received September 29, 1988

Revision received December 12, 1989

Accepted January 18, 1990 